\title{
EMOTION RECOGNITION FROM FACIAL EXPRESSION BASED ON BEZIER CURVE
}

\author{
Shruti Bansal ${ }^{1}$ and Pravin Nagar ${ }^{2}$ \\ ${ }^{1}$ Department of Computer Science ,Pranveer Singh Institute of Technology, Kanpur, \\ Uttar Pradesh \\ ${ }^{2}$ Assistant Professor Pranveer Singh Institute of Technology, Kanpur, Uttar Pradesh
}

\begin{abstract}
Human emotions are conveyed by different medium such as behaviours, actions, poses, facial expressions and speech. Multitudinous researches have been carried out to find out the relation between these mediums and emotions. This paper proposes a system which automatically recognizes the emotion represented on a face. Thus, a Bezier curve based solution together with image processing is used in classifying the emotions. Coloured face images are given as input to the system. Then, Image processing based feature point extraction method is applied to extract a set of selected feature points. Finally, extracted features like eyes and mouth, obtained after processing is given as input to the curve algorithm to recognize the emotion contained. Experimental results show average $60 \%$ of success to analyse and recognize emotion detection.
\end{abstract}

\section{KEYWORDS}

Emotion Recognition, Feature detection, Bezier Curve.

\section{INTRODUCTION}

In Biometric research, Recognition and analysis of human facial expression is very popular topic. There have been numerous researches done for this in each area of science.

Facial expressions are a way to express our emotions and also to provide gestures during social interactions. Basically, Researches have shown that facial expressions can significantly alter the meaning of what is spoken as well as control the flow of a conversation. Scrutinizing of emotions by computers has been a research area since Charles Darwin's age. In today's era, researches focus on improving human-computer interfaces, as well as on improving the actions which computer takes on feedback from the user. When computers could detect these emotional inputs, they would give specific and appropriate help to others in ways that are more compatible with the user's needs and preferences. It doesn't deal with the rest of the body, but only the facial features. Many techniques have been proposed for emotion detection, but not all give an accurate result or are easy to implement.

This paper proposes a scheme in which we adopted the feature based approach, i.e. automatically detection of eye and mouth from a still image is done using the Canny Edge detection algorithm and then the classification of emotion is conducted by implementing Bezier curve algorithms and measuring distance between certain points. The implementation of this algorithm is done on net beans API.

This paper is planned as follows next section reviews related works about emotion recognition through facial expression analysis. Section 3 is about the proposed system that uses two main 
steps to detect and recognize the facial emotion from still images. Experimental results are shown in section 4 , and section 5 is presenting about the conclusion.

\section{RELATED WORKS}

The inceptions of facial expression analysis began from the nineteenth century, when Darwin initially proposed the idea of general outward appearances in living creatures. Since the mid1970s, Ekman and Friesen (1975) have performed different investigations of human outward appearances, giving confirmation to bolster this all-inclusiveness hypothesis.

Ira et al proposed construction modeling of Hidden Markov Models for programming fragment and perceive human outward appearances from video arrangements. At that point a paper was proposed about programmed influence dissect and acknowledgment of human feeling. Investigates on perceiving feeling through outward appearance was proposed by Ekman, who began their work from the mental point of view. A strategy for an outward appearance acknowledgment for a human speaker by utilizing warm picture preparing and a discourse acknowledgment framework was explored by Yashnari. Spiro's et al recommended the extraction of proper facial elements and the ensuing acknowledgment of the client's passionate state that could be vigorous to outward appearance varieties among changed clients. Facial Emotion Recognition System for AUTISM was executed by Anjana R.besides, Lavanya M. Henry A. Rowley, Shumeet Baluja, Takeo Kanade in 1998 spearheaded Neural Network-Based Face Detection. All systems to investigate feelings are unique in relation to one another.

\section{PROPOSED METHOD}

This paper is about the implementation of face detection and emotion recognition. The proposed technique is made out of two noteworthy steps: the initial step is the location and examination of the elements of the data to the following step, and the second step is the acknowledgment of the facial feeling of the trademark highlights.

Piece outlines of our proposed technique are delineated here in figure 1 and figure 2 which demonstrates the flowchart of step 1 and step 2 individually.

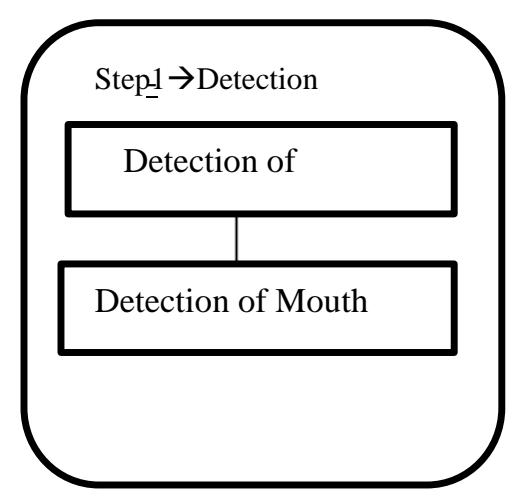

Figure 1 


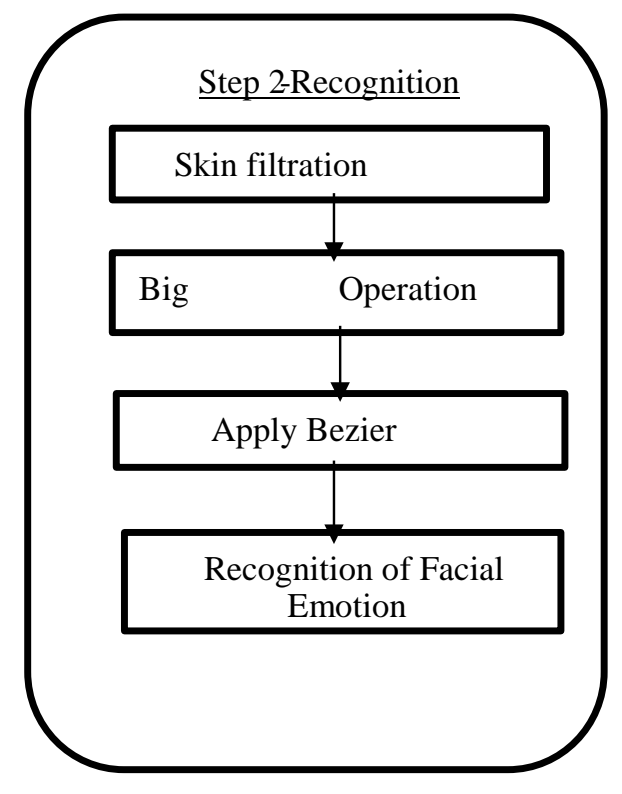

Figure 2

\section{The Flowchart of Proposed System}

In the initial step for detection, our techniques find and distinguish the locale of eye and mouth from a face and crop them. Along these lines, our proposed calculation for extraction of facial area for eye and mouth is done by Canny Edge Detection. At that point, skin filtration is connected, i.e. Identification of Skin Color utilizing $\mathrm{YCbCr}$ to the region of interest. After this, apply big connected Operation with the goal that we might ready to concentrate focuses to apply a Bezier bend to eyes and mouth. Finally, comprehension and acknowledgment of facial measuring so as to feel are performed the distinction between separation focuses in Bezier bend put away in the database..

\subsection{Eye and Mouth Detection}

We endeavor to separate the locale of eye and mouth from a still picture utilizing Canny Edge Detection calculation that was produced by John F. Watchful in 1986. The Canny Edge Location calculation was utilized through OpenCv API as a part of which haarcascades record, for eyes and mouth was predefined. By applying this algorithm, a red shading edge is drawn which demonstrate the part of eye and mouth being removed for further applying procedure to it. Figure 3 demonstrates the extraction of eyes and Figure 4 demonstrates the extraction of mouth.

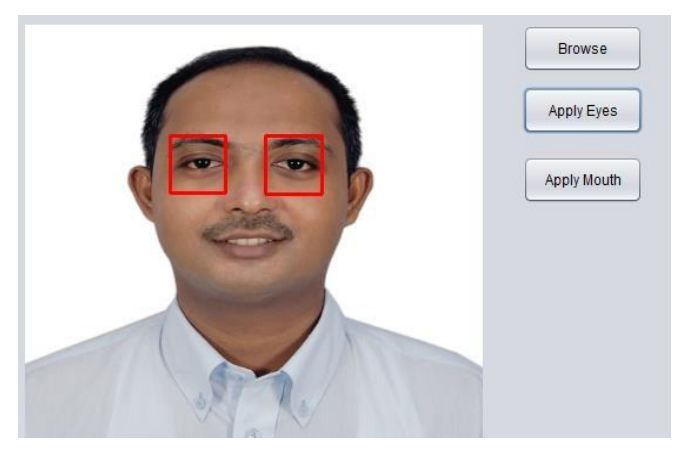

Figure 3. Detection of Eyes 


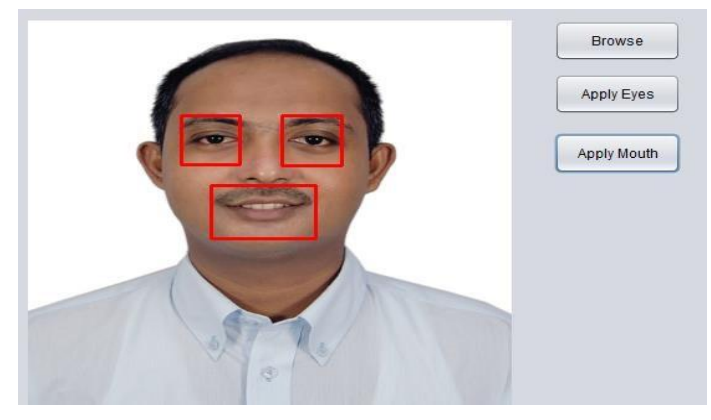

Figure 4. Detection of Mouth

\subsection{Detection of Skin Color Using Ycbcr}

The Color space changes and lighting pay is performed next. For this situation, we have adjusted to use a skin shading based methodology utilizing $\mathrm{YCbCr}$ model. With shading model, Y, a solitary part speaks to luminance data and shading data is put away as two shading contrast segment, $\mathrm{Cb}$ and $\mathrm{Cr}$. Part $\mathrm{Cb}$ is the contrast between the blue segment and a reference quality, and segment $\mathrm{Cr}$ is the distinction between the red segment and a reference esteem. Subsequent to changing over shading display, an enlightenment adjustments essential amid the pre-handling for the precise face discovery. Since the light condition is a critical component to impact on the execution of recognition, we endeavor preprocessing to balance the force esteem in a picture. Fundamentally, in skin filtration approach the skin shade of the given element is being separated and just those part is noticeable that is not in skin shading. Subsequently, figure 5 delineates the skin filtration of right eye.

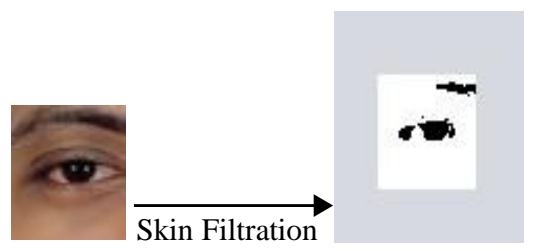

Figure 5

\subsection{Big Connect Operation}

Presently, in the skin filtrate picture, there are dark areas on the eye, and it might be conceivable that some other part additionally have somewhat diverse shading than skin shading. At that point we apply big connect district for discovering the dark area, which contains eye in the picture. Furthermore, we are certain that the enormous area is the eye on the grounds i.e. in the eye box, the eye is the biggest thing which is not the same as the skin.

In figure 6, it demonstrates that applying big connect interfaces operation to skin separated eye lead to the disposal of minor part and the outcome is the significant segment of the eye.

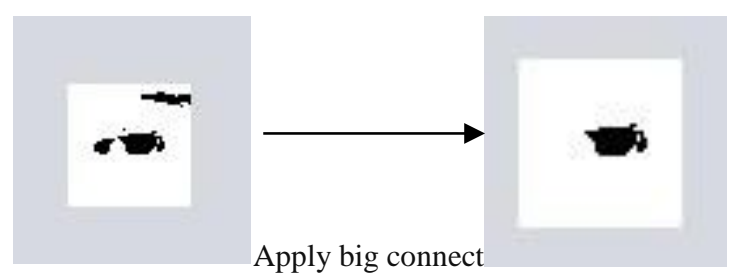

Figure 6 


\subsection{Bezier Curve}

We need to apply bend calculation on the resultant picture i.e. eye. For applying Bezier bend, we locate the beginning and completion pixel of the eye in flat. At that point we draw two digressions on upper eye from the beginning and completion pixel furthermore discover three focuses on the digression which is not the piece of the eye. For the lower eye, we discover three focuses like upper eye. We utilize Cubic Bezier bends to draw the Bezier bend of the eye. Along these lines, we draw two Bezier bends for the eye, one for upper eye and one for lower eye.

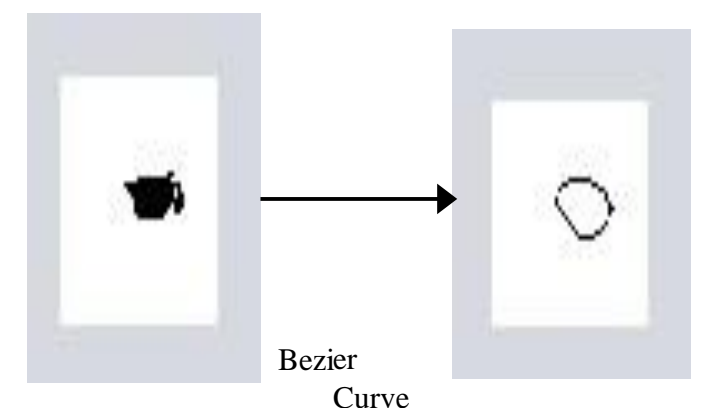

Figure 7

\subsection{Training Dataset}

In preparing database, there are two tables which are putting away individual data and files of four emotions with his/her own bends of outward appearance investigation. For discovery of emotion, we have to process the separation of coordinated correspondence of every interest areas between an information picture and the pictures in the preparation database. The Bezier curves are drawn over vital lines of removing components.

\section{These are the preparation sets:}

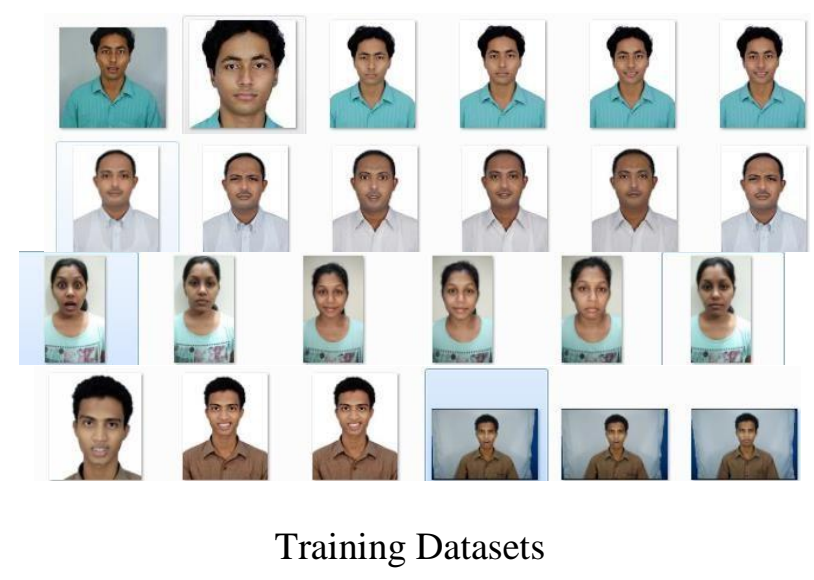

\section{EXPERIMENT AND RESULTS}

The expressions smile, sad, surprise and normal are considered in the implementation of the testing of face recognition. The faces with expressions are compared against the training face database. The Bezier points are interposed over the principal lines of facial features. These points for each curve form the adjacent curve segments. The distance is calculated based on the curve segments. Then, considerate and judgment of facial emotion are selected by determining 
similarity in faces. To categorize facial emotion, firstly we need to determine the expressions, i.e. Detection and Recognition of Facial. All images are colorful. Figure 5 shows sample facial images which expresses an emotion, for example, by neutral and smiling expression. The algorithm presented in the previous section is implemented with Java, and experiments are performed on Intel Core 2 Duo CPU, i.e. $2.00 \mathrm{GHz}$ PC with 2 GB RAM, as shown in Figure 8. The experiment shows the recognition results under different facial expressions such as smile, sad, surprise and normal. The proposed method gives successful emotion recognition of ratio $60 \%$. Experimental result tells that success ratio is better for smile, because the control points and curves for smile are more perfect. Grayscale images from the database are tough to be detected since the skin color pattern of facial region cannot be filtered in the image.

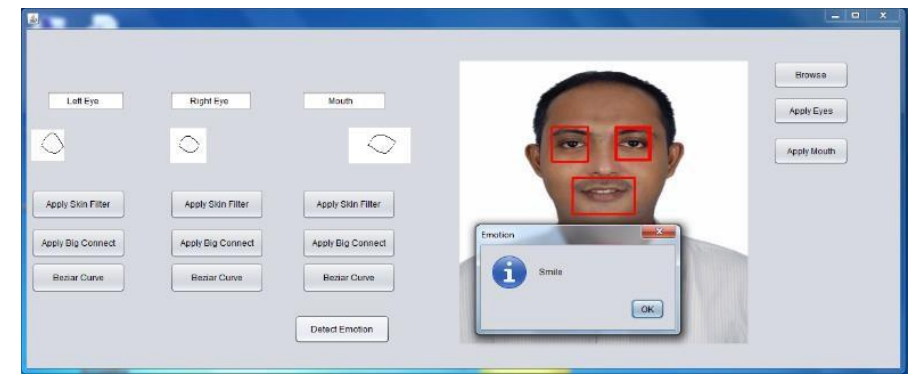

Figure 8

\section{CONCLUSION}

This work tries to resolve the problem of emotion recognition from an image based and a Bezier curve based approach. Various ways have been established to address the problem. In this approach performance was main issue. Thus, to increase performance of the system Bezier curve, which has to be drawn is made by taking 6 points from featured image. Emotion recognition is still a complex problem in the science field. Emotions can be expressed in different ways, i.e. facial expressions, pose, behavior, actions and speech. Facial expressions were the feature we acquired for the recognition of emotions in humans. Based on the points within the extracted feature a curve was drawn to identify the emotions contained. The proposed system is performance wise very good and provides better accuracy in emotion recognition. It has ability in recognizing the correct emotion with $70 \%$ accuracy even when the features are incorrectly identified.

\section{REFRENCES}

[1] Marian Stewart Bartlett, Gwen Littlewort, Ian Fasel, and Javier R. Movellan.(2003) Real time face detection and facial expression recognition: Development and applications to human computer interaction. In Proceeding of the Conference on Computer Vision and Pattern Recognition Workshop, 2003.

[2] Jeffrey F. CohnBy Ying-li Tian, Takeo Kanade. Recognizing action units for facial expression analysis. In 2000 IEEE Computer Society Conference on Computer Vision and Pattern Recognition (CVPR'00) - Volume 1, June 2000.

[3] a) Hehua Chi Lianhua Chi, b) Meng Fang a, c) Juebo Wuca FACIAL EXPRESSION RECOGNITION BASED ON CLOUD MODEL

a)International School of Software, Wuhan University, Wuhan

b)School of Computer Science and Technology, Huazhong

c)State Key Laboratory of Information Engineering in Surveying, Mapping and Remote Sensing, Wuhan University, Wuhan

[4] Spiros V. Ioannou, Amaryllis T. Raouzaiou, Vasilis A. Tzouvaras, Theofilos P. Mailis, Kostas C. Karpouzis, Stefanos D. Kollias* Emotion recognition through facial expression analysis based on a 
neurofuzzy network Image, Video and Multimedia Systems Laboratory, School of Electrical and Computer Engineering, National Technical University of Athens, Zografou 15773, received 24 March 2005; accepted 27 March 2005

[5] 1Anjana R., 2Lavanya M. Facial Emotions Recognition System for Autism International Journal of Advanced Engineering Technology E-ISSN 0976-3945 Int J Adv Engg Tech/Vol. V/Issue II/AprilJune, 2014/40-43 Research Paper

[6] Ashish Lonare1, Shweta V. Jain2 A Survey on Facial Expression Analysis for Emotion Recognition Student M.tech, Department of Computer Science and Engineering, Shri Ramdeobaba College of Engineering and Management, Nagpur, India. International Journal of Advanced Research in Computer and Communication Engineering Vol. 2, Issue 12, December 2013

[7] Akanksha Manuj ,Supriya Agrawal Automated Human Facial Expression and Emotion Detection: A Review Computer Science Department, Student. NMIMS University, India. International Journal of Computer Applications (0975 - 8887) Volume 110 - No. 2, January 2015

[8] Matthew S. Ratliff Department of Computer Science University of North Carolina Wilmington, Eric Patterson Department of Computer Science University of North Carolina Wilmington .Emotion Recognition Using Facial Expressions with Active Appearance Models.

[9] M.Swathi P.A Ashoka Vardini T.Bharat Kumar Department of CSE, JNTUA Department of CSE, JNTUA Software Engineer TCS, Chennai. Facial Expression Recognition Based on Co-ordinate and Bezier Curves. International Journal of Advanced Research in Computer Science and Software Engineering Research Paper.Volume 3, Issue 9, September 2013 ISSN: 2277 128X

[10] Yong-Hwan Lee Dept. of Applied Computer Engineering Dankook University, Yongin, Korea Woori Han and Youngseop Kim Dept. of Electronics Engineering Dankook University Cheonan, Korea. Emotional Recognition from Facial Expression Analysis using Bezier Curve Fitting. 2013 16th International Conference on Network-Based Information Systems.

[11] Jadhav, Jinal B. Mashruwala, Aditi K. Nehete, Pooja A. Panjari Real Time Emotion Recognition through Facial Expressions for Desktop Devices International Journal of Emerging Science and Engineering (IJESE) ISSN: 2319-6378, Volume-1, Issue-7, May 2013. 